\title{
Inteligencia de negocios en la banca nacional: Un enfoque basado en herramientas analíticas
}

\author{
Roo Huerta, Arlenys* \\ Boscán Romero, Neida**
}

\section{Resumen}

Desde el punto de vista tecnológico, la inteligencia de negocios implica el proceso de conversión de datos relevantes en información útil para la toma de decisiones estratégicas. El objetivo central del presente artículo es analizar la inteligencia de negocios en la banca nacional, desde la perspectiva de las herramientas analíticas, representadas por los servicios de transformación de datos, los procesos de análisis en línea, los servicios de explotación y el almacén de datos. Este estudio se basa en una metodología descriptiva, con un diseño de campo, se emplea la estadística descriptiva para el análisis de los resultados. Los hallazgos encontrados indican que las herramientas utilizadas con mayor frecuencia son los servicios de explotación, teniendo una notoria debilidad en la definición de los procesos realizados. Esto genera un gran impacto en la toma de decisiones, para proporcionar respuestas a interrogantes del negocio. Se concluye el uso de la inteligencia de negocios como un sistema de apoyo a los procesos llevados a cabo en las instituciones estudiadas.

Palabras clave: Inteligencia de negocios, toma de decisiones, herramientas analíticas, banca nacional.

\section{Business Intelligence in the National Bank: An Approach Based on Analytical Tools}

\begin{abstract}
From the technological viewpoint, business intelligence implies the process of converting relevant data into useful information for making strategic decisions. The central objective of this article is to analyze business intelligence in national banking, from the perspective of analytical tools repre-

Recibido: 05-12-11. Aceptado: 22-06-12

* Magister en gerencia de proyectos de investigación y desarrollo. Analista de tecnología del Banco Occidental de Descuento. Maracaibo, estado Zulia. Venezuela. e-mail: arlenysroo@ gmail.com

** Profesora titular de la Universidad Rafael Belloso Chacín, coordinadora de la línea de gerencia de la tecnología. Maracaibo, estado Zulia. Venezuela. e-mail: nboscan@urbe.edu.ve
\end{abstract}


sented by data transformation services, online analysis processes, exploitation services and data storage. This study is based on descriptive methodology with a field design and uses descriptive statistics to analyze the results. Findings indicate that the most frequently used tools are exploitation services, with a notable weakness in defining the processes performed. This generates a huge impact on decision making in terms of offering answers to questions about the business. The conclusion is to use business intelligence as a support system for the processes carried out in the institutions under study.

Key words: Business intelligence, decision making, analytical tools, national bank.

\section{Introducción}

A nivel mundial, la competencia en la industria financiera es cada día mayor, comprende una lucha constante dirigida a brindar productos y servicios acordes a las necesidades actuales de los clientes, lo cual alude a un proceso complejo que engloba recursos físicos, económicos y humanos, en acción conjunta con los cambios tecnológicos acelerados.

Considerando esta situación, el proceso de toma de decisiones se ha convertido en un elemento variable en función de las necesidades inmediatas de la organización, aspecto que repercute de forma directa en las acciones futuras que pueden incidir en el logro de los objetivos estratégicos de la misma.

El presente artículo tiene como objetivo general analizar la inteligencia de negocios en las instituciones que conforman la banca nacional a partir de sus herramientas, como proceso vinculante del factor humano y la tecnología, a través del uso de información de negocio de forma oportuna y confiable.

En este sentido, fueron consideradas las herramientas analíticas, con el objeto de identificar las herramientas de la inteligencia de negocios en la banca nacional, partiendo desde el punto de vista teórico, para luego contrastarlas con la realidad objeto de la investigación y ofrecer una alternativa a las organizaciones en el área financiera, empleando la inteligencia de negocios en la banca nacional para la adecuada toma de decisiones y el logro de los objetivos estratégicos.

El artículo se desarrolló en dos fases: la primera fue de tipo descriptiva, la cual permitió la definición desde el punto de vista teórico de la variable objeto de estudio; y una segunda fase de tipo analítica, a través del uso de la estadística descriptiva, con el fin de visualizar la frecuencia absoluta y relativa, así como la media y la desviación estándar para cada uno de los resultados. Se precisó una muestra no probabilística, representada por el personal a nivel supervisorio de cinco (5) instituciones pertenecientes al Sistema Bancario Nacional, seleccionado de forma intencional debido a la facilidad de acceso a la información de estudio, así como la experiencia de los seleccionados en la materia objeto de estudio. Se utilizó un instrumento con una escala de actitudes con cinco alternativas de respuesta: totalmente de acuerdo (TDA), de acuerdo (DA), ni de acuerdo ni en desacuerdo (NA/ND), en desacuerdo (ED) y totalmente en desacuerdo (TED).

Para el análisis de las medidas de tendencia central, se realizó un baremo con los diferentes niveles de confiabilidad 
Inteligencia de negocios en la banca nacional: Un enfoque basado en herramientas... Roo Huerta, Arlenys y Boscán Romero, Neida

y dispersión. Se calcula la desviación estándar para indicar el grado de dispersión de las respuestas obtenidas.

Se realizó un análisis comparativo a través de la técnica de la triangulación, donde se evidencia la observación directa y la encuesta, en contraste con el criterio de los autores, para cada una de las alternativas disponibles frente a las respuestas obtenidas, comprobando su relación con lo que se esperaba lograr.

Con los resultados obtenidos, el artículo pretende proveer a las instituciones financieras un instrumento que propicia la calidad funcional de los procesos organizacionales, en especial aquellos ligados a la toma de decisiones estratégicas, los cuales son vitales para el logro de los objetivos organizacionales, sirviendo de base para el desarrollo competitivo de las instituciones que integran la banca nacional.

\section{Inteligencia de Negocios: fundamentos}

La creciente demanda de información organizacional, ha originado la creación de nuevas herramientas y procesos acordes al ritmo cambiante y desmesurado que lo caracteriza, siendo la toma de decisiones un aspecto relevante dentro de la operación de toda empresa.

Partiendo de esta premisa, surge el término inteligencia de negocios (business intelligence- $B /$ ), expresión que hace referencia al proceso de toma de decisiones en función del rendimiento organizacional, convirtiendo simples datos en información relevante para los diversos niveles, orientándose en primera instancia al área estratégica organizacional (Vitt et al., 2003:11).
Esta tecnología, hace posible la gestión de información tanto organizada como no estructurada, resultante de la sinergia del procesamiento de datos y el uso de herramientas analíticas que fusionan la gestión de la información y del conocimiento, convirtiéndose en una ventaja altamente competitiva a nivel organizacional (Oramas, 2009:43).

La inteligencia de negocios se representa como un proceso que permite dar una rápida y efectiva respuesta ante interrogantes del negocio, convirtiendo volúmenes de datos en información útil para la gestión, control y administración estratégica.

A su vez, esta tecnología se encuentra enfocada en la administración y creación del conocimiento, que considera como base el conjunto de estrategias y herramientas analíticas, teniendo en común las siguientes características:

1. Apoyo a la toma de decisiones por medio de herramientas de análisis que permitan manipular, utilizar $y$ analizar la información de interés organizacional.

2. Transformación de los datos en información útil para la toma de decisiones.

3. Utilización de un método razonable para la administración y gestión del negocio.

Lo antes señalado permite definir la inteligencia de negocios como un término caracterizado por una amplia variedad de tecnologías, plataformas de software, especificaciones de aplicaciones y procesos, cuyo objetivo fundamental es contribuir a tomar decisiones que mejoren el desempeño de la organización y promueva su ventaja competitiva en el mercado. 
La inteligencia de negocios definida por Peña (2006:7) como un conjunto de aplicaciones y elementos gerenciales, faculta a la organización a tomar mejores decisiones de forma rápida y acertada, mediante la conversión de datos en información que permita establecer un puente entre las grandes cantidades de datos y la información requerida cotidianamente por quienes son los responsables del proceso de toma de decisiones.

Desagregando las definiciones antes mencionadas, se precisan los siguientes elementos como componentes de la inteligencia de negocios:

- Exploración de datos: permite descifrar patrones y tendencias entre los datos manejados en las diversas fuentes, generalmente de origen transaccional, además de descubrir nuevas relaciones entre los elementos involucrados en el proceso desconocidas hasta el momento.

- Anticipo a nuevas oportunidades: a través de la comprensión de la situación actual y futura del negocio, desarrollando diversos tipos de análisis a partir de la información organizacional que sirvan de apoyo a la toma de decisiones estratégicas, tácticas y operacionales para la ejecución de acciones que incrementen el desempeño de la organización.

- Procesos de análisis: se centra en la información resultante del procesamiento de datos, permitiendo su análisis como un todo en lugar de visualizarlos como simples datos aislados e independientes.

- Generación de valor a las áreas de negocio: provee de elementos tecnológicos que generan un mayor valor en las áreas de negocio existentes, incluyendo de forma implícita una reducción de costos e incremento de los márgenes de utilidad.

Por otra parte, este término es asociado con un ambiente que facilita la toma de decisiones a través del uso de las tecnologías de la información para el descubrimiento de nuevas oportunidades, habilitando el pensamiento y accionar estratégicos (Hurtado, 2008:57).

Se puede destacar que toda actividad inmersa en la inteligencia de negocios, debe estar considerada como un modelo integral que permita la obtención de los resultados deseados, de acuerdo a la toma de decisiones estratégicas, lo cual repercute en el logro de los objetivos de toda organización, indiferentemente de la naturaleza dentro de la cual se encuentre enmarcada.

Otros conceptos de inteligencia de negocios, señalan que está vinculada con aplicaciones no convencionales, que se han convertido en punto focal en las organizaciones que se encuentran encaminadas a la adopción de tecnologías que van más allá de la integración de elementos de hardware y software y que involucran el conocimiento, donde su implementación es vista como un ciclo evolutivo a través del tiempo (Olszak y Ziemba, 2012:136).

En este sentido, los procesos modernos han migrado su paradigma, teniendo como principal recurso el conocimiento. Este conocimiento, en sus distintas formas de expresión, puede sustituir cualquier factor tradicional de producción, generando nuevas alternativas tecnológicas basadas en conocimiento, convirtiéndose en un elemento esencial para la toma de decisiones.

La inteligencia de negocios, hace referencia al análisis de los procesos de negocio, para la carga de información en 
Inteligencia de negocios en la banca nacional: Un enfoque basado en herramientas... Roo Huerta, Arlenys y Boscán Romero, Neida

bases de datos, generalmente conocidas como data warehouse (DWH), lo cual permite crear un entorno en el que los procesos pueden ser cuidadosamente monitoreados mediante herramientas analíticas orientadas al desarrollo de reportes y tableros de control que simplifican la producción de dicho proceso (Plant y Murrell, 2007).

De igual forma facilita el proceso de transformación y mejora de las organizaciones, a través del uso de nuevos enfoques y paradigmas tecnológicos, donde el valor reside en las personas.

Es por ello que, dentro de la arquitectura de las herramientas de inteligencia de negocios, es necesario destacar el papel de la administración del conocimiento; aspecto que considera de forma implícita tres elementos clave: los datos, la información y el conocimiento. La efectividad en el uso de estos componentes, así como la rápida aplicación de los mismos, constituye una ventaja sostenible para las aplicaciones empleadas en cualquier organización (Peña, 2006).

De esta forma, se observan diferentes puntos de vistas asociados a la inteligencia de negocios, de acuerdo a los enfoques señalados anteriormente, teniendo en común el conocimiento como principal factor productivo para el desarrollo de herramientas analíticas, del cual depende el resto de los recursos organizacionales.

En síntesis, la inteligencia de negocios ofrece un apoyo tecnológico a través de plataformas, especificaciones de aplicaciones y procesos, destinados a la toma de decisiones con una mejor calidad y rapidez en las respuestas, en conjunto con el manejo de la administración del conocimiento, dando lugar a la solu- ción de problemas presentados en cuanto al manejo de indicadores, establecimiento de metas y cumplimiento de objetivos estratégicos dentro de las instituciones que desean aumentar su competitividad y eficiencia, brindando respuestas efectivas en un dominio concreto a partir de un enfoque estratégico.

\section{Teorías de las herramientas de la inteligencia de negocios}

Desde la perspectiva tecnológica, la arquitectura de la inteligencia de negocios cuenta con gran número de recursos que conforman el marco de esta tecnología, donde las herramientas analíticas han logrado una acelerada penetración en el mercado, debido a que las empresas buscan nuevas soluciones que les permitan liderar de forma ágil, confiable y eficiente, facilitando el procesamiento de grandes volúmenes de información en tiempo real, para la toma de decisiones y la gestión diaria del negocio.

Peña (2006:31) precisa una serie de herramientas analíticas dentro del marco conceptual de la inteligencia de negocios. De esta forma, un almacén de datos conocido comúnmente como data warehouse (DWH), es un paradigma para la organización, análisis y proyección de datos, con el propósito de brindar una infraestructura tecnológica de información útil a la toma de decisiones en una organización, originado como consecuencia del gran volumen de transacciones realizadas, pero con poca capacidad de análisis al disponer de reportes específicos y consultas interactivas.

Este elemento integra el ambiente del depósito de datos, el cual posee dife- 
rentes temas que pueden ser analizados independientes o de manera integrada, además de proveer un conjunto de instrumentos que permiten consultar, analizar y presentar información.

De igual manera, Pineda y Díaz (2006:11) definen este término como un repositorio donde se integran diferentes fuentes de información, necesaria para soportar los procesos de toma de decisiones. Constituye el motor de las soluciones de inteligencia de negocios, por lo que facilita la interpretación de la información relevante para el negocio y soporta el proceso de toma de decisiones.

Un DWH es un repositorio de recuperación y consolidación de información de forma periódica, proveniente de diversas fuentes, los cuales son dimensionados y normalizados en una estructura, generalmente actualizada por lotes, a diferencia de otros elementos que son actualizados cada vez que una transacción es registrada en el sistema origen (Rainardi, 2007:1).

Del análisis de los criterios anteriores, se tiene que este concepto sociotecnológico se caracteriza por la colección de datos orientados a temas integrados, organizados en una estructura física, establecida como un objeto de almacenamiento para la posterior explotación analítica y de gestión en función de las necesidades empresariales, cuyo objetivo principal es unificar en un mismo lugar la base de datos y el conjunto de modelos que sostienen los procesos transaccionales, de tal forma que permita una acertada toma de decisiones.

Aunado a la definición del almacén de datos, se encuentra el término de tienda de datos o data mart. Peña (2006:31) establece que una tienda de datos realiza una labor similar a un almacén, con la particularidad de que éste se encuentra integrado por la información de una función o área específica, volumen de datos limitado y con un alcance menor que un $\mathrm{DWH}$, generalmente conformado por información procedente de varios sistemas transaccionales consolidados en un repositorio común.

Otra de las herramientas analíticas más destacas es el repositorio de metadatos, el cual representa una estructura dotada de los mecanismos necesarios para el almacenamiento, acceso y administración de la información depositada en el DWH, acorde a los procedimientos y aplicaciones que conforman los modelos de información (Peña, 2006:32).

Existen dos tipos de metadatos clasificados de acuerdo a la disposición de los mismos; en primer lugar los técnicos, orientados a la creación, mantenimiento y manejo del DWH; y en segundo lugar los del negocio, destinados a brindar diversas perspectivas de la información ubicada en el almacén para la elaboración de consultas, reportes, evaluaciones y, en consecuencia, simplificando el proceso de toma de decisiones.

Los metadatos, constituyen una base de datos integrada por información relacionada con la estructura de los datos, el significado y uso de la data, así como las reglas y demás información asociada, representando una infraestructura común de almacenamiento (Rainardi, 2007).

Estos conceptos, precisan a esta herramienta analítica como una estructura contenedora de un conjunto de datos que capturan la lógica del negocio, utilizada para la construcción de las dimensio- 
Inteligencia de negocios en la banca nacional: Un enfoque basado en herramientas... Roo Huerta, Arlenys y Boscán Romero, Neida

nes y medidas de los modelos de inteligencia de negocios.

De esta forma, los repositorios de metadatos constituyen una parte integral de los sistemas de toma de decisión, creados de forma conjunta con el DWH y tiendas de datos, que forman parte de las herramientas analíticas de la inteligencia de negocios.

Por otra parte, los servicios de transformación de datos (data transformation services - DTS), constituyen funciones de acceso a fuentes de datos por medio de búsquedas, extracción, conversión y almacenamiento. Estos servicios, provienen de la fuente denominada "datos primarios" hacia un modelo de almacenamiento, encargándose de su importación, validación, transformación, integración, almacenamiento y actualización. Este elemento varía en cada organización, ya que depende de forma directa del proveedor de servicios seleccionado para tal fin.

Los DTS emplean diversos elementos orientados en la extracción de los datos, capaces de explotar plataformas de computadora, sistemas operativos, marcas de manejadores de base de datos y ambientes, cuya ejecución es programada previamente en una tarea (Peña, 2006:33).

De igual manera, representan un conjunto de utilidades gráficas, aplicaciones y objetos programables, comprendiendo los procesos que van desde la extracción hasta la carga final de información de negocio, útil para la toma de decisiones (Lluís, 2007).

De los juicios anteriores se puede concluir que un DTS es una serie de funciones destinadas a la extracción, transformación y carga de datos en un DWH. La extracción hace referencia a la trans- ferencia de datos desde el sistema origen al DWH; por su parte la transformación garantiza que la información contenida en las tiendas de datos maneje un sistema estándar de códigos y abreviaturas, para posteriormente realizar la carga de los datos en el repositorio establecido.

De igual manera, los procesos de análisis en línea (on-line analytical processing-OLAP), constituyen un ambiente de proceso equivalente a las bases de datos y almacenamiento de datos, destinado a la recolección, organización y presentación de la información de acuerdo a modelos predefinidos que faciliten su explotación.

Para ello, se requiere de un motor similar a los manejadores de base de datos (servidor OLAP), encargado de brindar una función de sistema de soporte a las decisiones, con capacidad de análisis, facilidades para compartir datos y una perspectiva multidimensional. Esta última, hace referencia a una estructura integrada por información de diferentes puntos de vista acerca de diversas áreas de negocio, que resultan vitales para el proceso de toma de decisión, a partir del análisis de datos que satisfacen los requerimientos de una amplia variedad de usuarios (Peña, 2006).

En síntesis, el ambiente OLAP se compone de estructuras de datos y motores encargados de su administración y explotación. El flujo de operación inicia con la extracción de la información registrada en el depósito para organizarla en un repositorio denominado almacén OLAP, a partir de la cual se producen las tablas transformadas, cuyo contenido y formato está adaptado con el propósito de facilitar la consulta realizada por parte del usuario final. 
Finalmente, los servicios de explotación, también conocido como servicios de extracción, transformación y carga (extract, transform and load - ETL), hacen referencia a una plataforma de procedimientos que aprovechan las facilidades que brinda un DWH, los servidores y tablas utilizadas para la explotación, modelado, evaluación y posterior toma de decisiones (Peña, 2006).

Un DWH permite trasladar datos desde múltiples fuentes, darles el formato apropiado, depurarlos y cargarlos en otra base de datos, facilitando el análisis de la información contenida en otros sistemas operacionales para apoyar un proceso del negocio. Este proceso se lleva a cabo por medio de herramientas analíticas utilizadas para cada una de sus etapas, que proporcionan la integración de datos ubicados en diferentes fuentes de información (Herrera, 2009).

Por su parte, Davis et al. (2008:142) definen un ETL como el conjunto de actividades que permiten trasladar los datos desde múltiples fuentes, realizar los cambios correspondientes al ajuste, transformación, adecuación y conversión de la información, para luego cargarlos en otra base de datos multidimensional y ser analizados, de tal forma que permita el apoyo a los procesos de negocio y a la toma de decisiones oportuna en los diversos niveles organizacionales.

Estos procesos, se encargan de consolidar la información de varias fuentes, los cuales tienen la capacidad de conectarse a los orígenes de datos y transformarlos para su posterior integración en un almacén de datos dimensional, donde se encuentra la información una vez establecidas las reglas del negocio para la realización del análisis útil para la toma de decisiones.

Alcántar (2012) establece una serie de beneficios a nivel organizacional que son provistos por las herramientas de la inteligencia de negocios, siendo los más relevantes los que se indican a continuación:

- Alineación organizacional a partir de la definición de los objetivos estratégicos.

- Desarrollo de análisis de desempeño de toda la empresa.

- Rápida y más informada toma de decisiones, incrementando su agilidad para dar respuesta a las demandas del mercado actual

- Visibilidad del comportamiento financiero de la organización.

- Aumento de la rentabilidad de la base de clientes.

Al analizar los conceptos emergentes de las herramientas de la inteligencia de negocios, resulta evidente que para la implantación de soluciones basadas en esta tecnología es imprescindible el conocimiento de cada uno de los elementos asociados tanto al entorno de desarrollo, como al análisis y transformación de los datos en información relevante para cualquier sector empresarial, incluyendo las organizaciones que ofrecen servicios financieros a sus clientes, tales como la banca nacional, para definir la herramienta que se adapta a sus necesidades y garantizar la calidad de la información. 
Inteligencia de negocios en la banca nacional: Un enfoque basado en herramientas... Roo Huerta, Arlenys y Boscán Romero, Neida

\section{Herramientas de la inteligencia de negocios: aplicabilidad en la banca nacional}

La necesidad de herramientas analíticas que faciliten la toma de decisiones, de acuerdo al grado de incertidumbre futura, se ha convertido en el punto clave de toda organización, en virtud de conocer cómo las diferentes decisiones afectan al plan estratégico previamente establecido. En este sentido, el cumplimiento de metas, las fluctuaciones de los costos financieros, así como el nivel de satisfacción de los clientes, tanto internos como externos, son aspectos que han motivado a las instituciones a incursionar en una nueva área: la inteligencia de negocios.

Partiendo de esta premisa, la Asociación Bancaria de Venezuela (2010), identifica dentro de las funciones básicas que deben ser realizadas por las herramientas de inteligencia de negocios en toda institución financiera, a la gerencia de riesgos y el procesamiento de información, a través de un correcto intercambio entre las áreas de riesgo de las instituciones del sistema, a fin de establecer un entorno homogéneo que apoye la toma de decisiones.

Dentro del contexto de la banca nacional, las herramientas de la inteligencia de negocios permiten implementar el uso de tecnología para la transformación efectiva de datos en información relevante, requerida principalmente para la toma de decisiones basadas en información correcta y certera, a fin de mejorar las operaciones de la banca, garantizando el cumplimiento de las normas y necesida- des de la organización (Banco Occidental de Descuento, 2012).

De acuerdo a estas teorías, el uso de las herramientas analíticas dentro del contexto financiero hace posible que la inteligencia de negocios exista en todos los niveles de la organización, transformando estas necesidades de información en resultados concretos, convirtiéndose en empresas competitivas ante las economías dinámicas que las caracterizan.

Por otra parte, las organizaciones actualmente recopilan y almacenan volúmenes inimaginables de información resultante de sus operaciones, por lo que las herramientas analíticas ofrecen a las empresas un marco para el empleo de esa información en una mejor toma de decisiones, a fin de extraer valoraciones que se transformen en una ventaja competitiva en la economía actual (Guanipa y Ramírez, 2011).

De esta forma, la tecnología que caracteriza a las herramientas de la inteligencia de negocios garantiza la accesibilidad a la información, a través de instrumentos analíticos que apoyen la toma de decisiones y, en consecuencia, generen mayor valor a las áreas de negocio existentes, anticipando nuevas oportunidades a partir de las cuales se generen ventajas altamente competitivas.

En este orden de ideas, en el contexto de la banca nacional, se ha identificado y analizado la situación actual de las herramientas de inteligencia de negocios, tomando como base la dimensión herramientas, integrada por una serie de indicadores que permitieron medir la dimensión previamente definida, los cuales se pueden observar en el Cuadro 1. 


Cuadro 1
Indicadores de la dimensión
herramientas de la inteligencia
de negocios

Fuente: Elaboración propia.

Para el análisis de las medidas de tendencia central, se realizó un baremo con los diferentes niveles de confiabilidad y dispersión. El valor de la media permitió la categorización de los ítems relacionados con el análisis de las herramientas de la inteligencia de negocios. Partiendo de este criterio, en la Tabla 1 se encuentra la escala de medición empleada para el análisis de la media.

Tabla 1

Escala de ponderación de la medida de tendencia central

\begin{tabular}{ccl}
\hline Rango & Intervalo & \multicolumn{1}{c}{ Categoría } \\
\hline 5 & $4.40-4.50$ & Muy Alto Nivel \\
4 & $4.29-4.39$ & Alto Nivel \\
3 & $4.18-4.28$ & Moderado Nivel \\
2 & $4.07-4.17$ & Bajo Nivel \\
1 & $3.954-06$ & Muy Bajo Nivel \\
\hline
\end{tabular}

Fuente: Elaboración propia.

Por su parte, el cálculo de la desviación estándar permitió indicar el grado de dispersión de las respuestas obtenidas. De acuerdo a clasificación, escala y rango establecido, representado por las puntuaciones manejadas para el desarrollo de la investigación, es decir, cinco
(5) y uno (1) respectivamente, lo cual permitió elaborar para su respectivo análisis la siguiente tabla de rango, intervalo y categoría (Tabla 2).

\begin{tabular}{|c|c|c|}
\hline \multicolumn{3}{|c|}{$\begin{array}{c}\text { Tabla } 2 \\
\text { Escala de ponderación de la } \\
\text { desviación estándar }\end{array}$} \\
\hline Rango & Intervalo & Categoría \\
\hline 5 & $0.74-0.78$ & Muy Alta Dispersión \\
\hline 4 & $0.69-0.73$ & Alta Dispersión \\
\hline 3 & $0.64-0.68$ & Moderada Dispersión \\
\hline 2 & $0.59-0.63$ & Baja Dispersión \\
\hline 1 & $0.53-0.58$ & Muy Baja Dispersión \\
\hline
\end{tabular}

Fuente: Elaboración propia.

Con respecto a la dimensión herramientas analíticas, se tiene que las instituciones analizadas, pertenecientes a la banca privada venezolana, ofrecen productos y servicios financieros a sus clientes. Resulta relevante que la alta gerencia cuente con herramientas tecnológicas que permitan la correcta y efectiva toma de decisiones, a fin de conocer la situación actual y futura de las mismas.

La identificación de las herramientas, se realizó a través de la evaluación de los indicadores que la definen: almacén de datos, repositorio de metadatos, servicios de transformación de datos, procesos de análisis en línea y los servicios de explotación.

En el Gráfico 1, se observa que el 90\% del personal mostró una actitud positiva ante el hecho de que las bases de datos y los modelos transaccionales se encuentran consolidados para facilitar el proceso de toma de decisión, en especial los provenientes de diversas fuentes, mientras que el $10 \%$ restante mantuvo 
Inteligencia de negocios en la banca nacional: Un enfoque basado en herramientas... Roo Huerta, Arlenys y Boscán Romero, Neida

Gráfico 1 Almacén de datos en la banca nacional

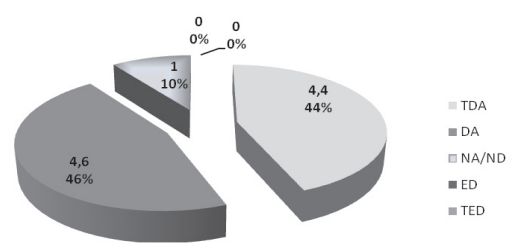

Fuente: Elaboración propia.

una postura indiferente ante los juicios asociados a los almacenes de datos. Esto demuestra que la información manejada incide de forma directa en el análisis multidimensional, el cual es realizado como producto de la consolidación de información de varios orígenes de datos de carácter transaccional.

El el Gráfico 2 se observa con el 97\% de los resultados dentro del repositorio de metadatos, los datos capturan la lógica del negocio a través de mecanismos de almacenamiento que forman parte de los modelos establecidos, diferenciándose de una tendencia neutra del 3\% del resto de los sujetos encuestados.

En lo que respecta al indicador servicios de transformación de datos, un $88 \%$ de la muestra seleccionada estuvo de

\section{Gráfico 2 \\ Repositorio de metadatos en la banca nacional}

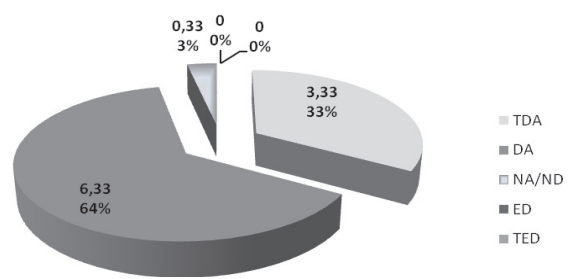

Fuente: Elaboración propia. acuerdo con el funcionamiento de los procesos de extracción, transformación y carga actual de datos, por lo que se encuentran satisfechos con los resultados originados a partir de estos procesos, mientras que el $12 \%$ restante mantuvo una postura neutral ante los juicios emitidos en relación a este indicador (Gráfico 3).

\section{Gráfico 3}

Servicios de transformación de datos en la banca nacional

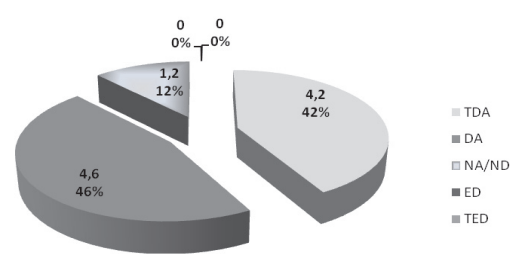

Fuente: Elaboración propia.

El indicador procesos de análisis en línea (Gráfico 4) evidencian que el mayor porcentaje, representado por el $94 \%$, mantuvo una tendencia positiva hacia la premisa de que el procesamiento analítico obedece a modelos predefinidos por las organizaciones, observando una baja tendencia del $6 \%$ que no estuvo de acuerdo ni en desacuerdo.

\section{Gráfico 4 \\ Procesos de análisis en línea}

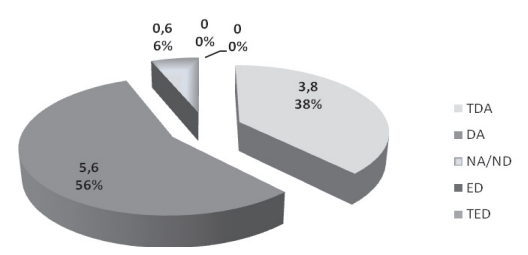

Fuente: Elaboración propia. 
Por último, los resultados indicados en el Gráfico 5 para el indicador servicios de explotación, evidencian que un $92 \%$ de la muestra estuvo de acuerdo que los procesos de explotación permiten aprovechar las facilidades de los modelos existentes, considerando la adecuada conexión a los orígenes de datos, en contraste con el $8 \%$ restante que se mantuvo neutral ante tal afirmación, por lo que la tendencia de las respuestas para este indicador fue positiva.

\section{Gráfico 5 Servicios de explotación}

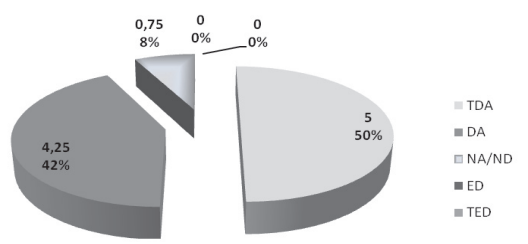

Fuente: Elaboración propia.

Según la escala empleada para el análisis de la medida de tendencia central de las herramientas de inteligencia de negocios, se obtuvo un promedio de 4.34, teniendo una predominancia por los servicios de explotación, posicionándola en un alto nivel de confiabilidad, tal como se refleja en el Diagrama 1.

En cuanto a la escala empleada para el análisis de la desviación estándar de la variable, el Diagrama 2 muestra los valores para cada uno de los indicadores que conforman las herramientas, obteniendo una puntuación de 0.62 con una baja dispersión, la cual indica que los resultados se encuentran cerca de la media, reflejando la consistencia de los datos obtenidos.

\section{Diagrama 1 Media de las herramientas}

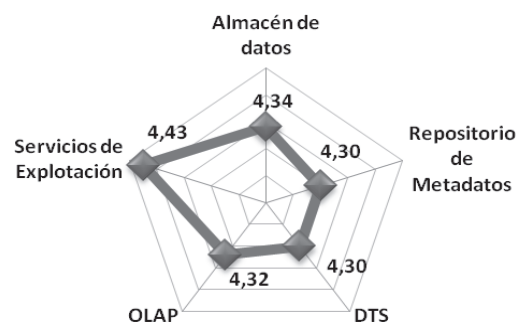

Fuente: Elaboración propia.

\section{Diagrama 2 Desviación estándar de las herramientas}

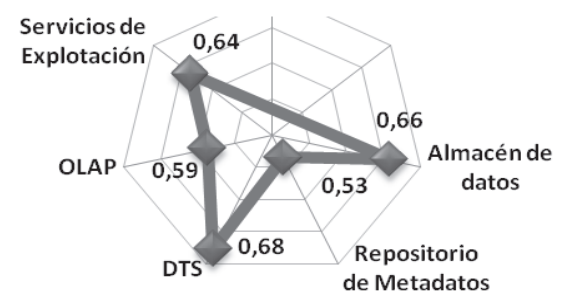

Fuente: Elaboración propia.

Los resultados evidencian que las herramientas señaladas son de gran relevancia dentro de los procesos organizacionales, en especial los servicios de explotación, ya que los mismos consideran la adecuada conexión a diversos orígenes de datos, al igual que la debida transformación de los mismos para su posterior carga en la estructura multidimensional.

Estas afirmaciones coinciden con la teoría avalada por Rainardi (2007), quien destaca la capacidad de la herra- 
Inteligencia de negocios en la banca nacional: Un enfoque basado en herramientas... Roo Huerta, Arlenys y Boscán Romero, Neida

mienta para conectarse a los orígenes de datos y transformarlos para su posterior integración, donde se encuentra la información, una vez establecidas las reglas de negocio para administrar y controlar las actividades dentro de cada una de sus unidades, con la finalidad de apoyar la toma de decisiones.

De igual manera, estas evidencias son compartidas por Peña (2006), ya que las organizaciones que constituyen el escenario de estudio aprovechan las facilidades de los almacenes de datos para la ejecución de los procesos de explotación, esenciales para la toma de decisiones y, por consiguiente, para el logro de los objetivos de las mismas.

Paralelo a la encuesta realizada, fue empleada la observación directa del entorno de trabajo, con lo cual se pudo evidenciar la situación actual presente en materia de inteligencia de negocios en la banca nacional.
En el Cuadro 4, se comparan los resultados obtenidos luego de la aplicación de los instrumentos utilizados, en conjunto con el juicio del investigador.

De esta forma, se evidencia que en la observación directa existen elementos que no fueron consistentes, en comparación con los resultados obtenidos en la encuesta, de acuerdo con la opinión del personal que forma parte de la labor en el área de inteligencia de negocios de las diversas instituciones de la banca nacional.

Para el indicador almacén de datos, se muestra una inadecuada organización de los datos, lo cual dificulta la realización del análisis multidimensional por parte del personal que labora en las instituciones de la banca nacional, seguido de los servicios de transformación de datos, donde se evidencia que los procesos de transformación no son llevados a cabo de forma correcta, originado por la inadecuada definición de los mismos.

\section{Cuadro 4. Comparación de resultados}

\begin{tabular}{|c|c|c|c|}
\hline Indicador & Observación Directa & Encuesta & Juicio del Investigador \\
\hline $\begin{array}{l}\text { Almacén } \\
\text { de datos }\end{array}$ & $\begin{array}{l}\text { Se consideran datos de } \\
\text { diversas fuentes, sin } \\
\text { embargo la información } \\
\text { manejada no responde a } \\
\text { una adecuada organiza- } \\
\text { ción de los datos, dificul- } \\
\text { tando el análisis multidi- } \\
\text { mensional. }\end{array}$ & $\begin{array}{l}\text { Se presenta la consoli- } \\
\text { dación de modelos que } \\
\text { agilizan el análisis de la } \\
\text { información para la toma } \\
\text { de decisiones. }\end{array}$ & $\begin{array}{l}\text { Los modelos creados } \\
\text { permiten una correcta y } \\
\text { eficiente toma de deci- } \\
\text { siones, pero debe consi- } \\
\text { derarse una adecuada } \\
\text { organización de los da- } \\
\text { tos. }\end{array}$ \\
\hline $\begin{array}{c}\text { Repositorio } \\
\text { de } \\
\text { metadatos }\end{array}$ & $\begin{array}{l}\text { Los mecanismos de al- } \\
\text { macenamiento obede- } \\
\text { cen a reglas en función a } \\
\text { la lógica del negocio, } \\
\text { siendo relevante el signi- } \\
\text { ficado de los datos. }\end{array}$ & $\begin{array}{l}\text { La lógica de negocio es } \\
\text { captada en los reposito- } \\
\text { rios utilizados, dándole } \\
\text { menor importancia al } \\
\text { significado de los datos. }\end{array}$ & $\begin{array}{l}\text { Es necesario considerar } \\
\text { el significado de la infor- } \\
\text { mación para determinar, } \\
\text { a partir de ésta, los datos } \\
\text { relevantes. }\end{array}$ \\
\hline
\end{tabular}




\section{Cuadro 4. Continuación}

\begin{tabular}{|c|c|c|c|}
\hline Indicador & Observación Directa & Encuesta & Juicio del Investigador \\
\hline $\begin{array}{l}\text { Servicios } \\
\text { de } \\
\text { transformación } \\
\text { de datos }\end{array}$ & $\begin{array}{l}\text { Los procesos de trans- } \\
\text { formación no siempre se } \\
\text { ejecutan de forma co- } \\
\text { rrecta, originado como } \\
\text { resultado de una inade- } \\
\text { cuada definición. }\end{array}$ & $\begin{array}{l}\text { Las funciones de extrac- } \\
\text { ción se encuentran pro- } \\
\text { gramadas, pero existe } \\
\text { una debilidad en la defi- } \\
\text { nición de los procesos. }\end{array}$ & $\begin{array}{l}\text { e requiere una mejor } \\
\text { definición de los proce- } \\
\text { sos, para mejorar los } \\
\text { servicios de transforma- } \\
\text { ción. }\end{array}$ \\
\hline $\begin{array}{l}\text { Procesos de } \\
\text { análisis en } \\
\text { línea }\end{array}$ & $\begin{array}{l}\text { Se utilizan modelos para } \\
\text { el análisis de datos, así } \\
\text { como mecanismos inte- } \\
\text { ractivos que facilitan la } \\
\text { toma de decisiones. }\end{array}$ & $\begin{array}{l}\text { El análisis interactivo y el } \\
\text { desarrollo de modelos } \\
\text { son adecuados, pero es } \\
\text { necesario considerar las } \\
\text { directrices del negocio. }\end{array}$ & $\begin{array}{l}\text { Las directrices del nego- } \\
\text { cio son las que deberían } \\
\text { determinar los modelos } \\
\text { a utilizar, de tal forma } \\
\text { que facilite el análisis de } \\
\text { los datos. }\end{array}$ \\
\hline $\begin{array}{l}\text { Servicios de } \\
\text { explotación }\end{array}$ & $\begin{array}{l}\text { Existe la presencia de } \\
\text { herramientas, pero no } \\
\text { son utilizadas en todas } \\
\text { las etapas del proceso, } \\
\text { aunado al hecho de que } \\
\text { se presentan inconve- } \\
\text { nientes en la transforma- } \\
\text { ción. }\end{array}$ & $\begin{array}{l}\text { Existe una debilidad en } \\
\text { cuanto a la definición de } \\
\text { las herramientas usadas } \\
\text { en cada etapa. }\end{array}$ & $\begin{array}{l}\text { Las herramientas usa- } \\
\text { das son vitales para las } \\
\text { actividades realizadas } \\
\text { en cada fase del proce- } \\
\text { so, por lo que deberían } \\
\text { darle una mayor relevan- } \\
\text { cia. }\end{array}$ \\
\hline
\end{tabular}

Fuente: Elaboración propia.

\section{Conclusiones}

Entre los hallazgos más importantes, se revela la necesidad de un cambio en el uso de tecnologías que permitan responder a las interrogantes de negocio; estas resultan decisivas para determinar acciones, tanto presentes como futuras, necesarias para el logro del éxito de las instituciones que conforman el Sistema Bancario Nacional.

Una de estas realidades, es el uso de las aplicaciones y mecanismos de control existentes, que permiten detectar las desviaciones y realizar las acciones necesarias para el cumplimiento de los objetivos organizacionales. Sin embargo, resulta notoria la falta de asignación de niveles de acceso para la manipulación de estas aplicaciones, al igual que la elaboración de planes de mantenimiento y actualización de las bases y almacenes de datos, a fin de estar en concordancia con la realidad cambiante que caracteriza a la banca nacional.

La correcta transformación de los datos manejados en las instituciones financieras, en función de las reglas de negocio previamente definidas, representa un elemento esencial para la toma de decisiones de forma rápida, precisa y asertiva, constituyendo el propósito fundamental de la inteligencia de negocios.

En lo que a herramientas de inteligencia de negocios se trata, los servicios de explotación proveen una adecuada 
Inteligencia de negocios en la banca nacional: Un enfoque basado en herramientas... Roo Huerta, Arlenys y Boscán Romero, Neida

conexión a las diversas fuentes de datos, generalmente de origen transaccional, a partir de las cuales es procesada la información que se encuentra contenida en las estructuras multidimensionales existentes.

La investigación confirma que las herramientas que se utilizan con mayor frecuencia dentro del proceso de inteligencia de negocio en la banca nacional son los servicios de explotación, los almacenes de datos y los procesos de análisis en línea, dejando de lado los servicios de transformación de datos y los repositorios de metadatos, siendo estos últimos los modelos de información que contemplan tanto los mecanismos de almacenamiento como la lógica del negocio, elementos sumamente relevantes para potenciar la adecuada y eficiente toma de decisión en la organización.

Se evidencia el uso de la inteligencia de negocios como un sistema de apoyo a los procesos llevados a cabo en las instituciones que integran la banca nacional, enmarcados en las necesidades y requerimientos establecidos por dichas instituciones, con la finalidad de aumentar su competitividad al mantener y fortalecer la confianza de sus clientes, a través de una adecuada toma de decisiones estratégicas.

\section{Referencias bibliográficas}

Alcántar, Ramón (2012). Por una organización más ágil con base en inteligencia de negocios. Revista Electrónica NTEK. Año 2012, Edición Enero 2012. México D.F., México.

Asociación Bancaria de Venezuela. (2010). Evolución del Sistema Financiero Venezolano. Federación Latinoame- ricana de Bancos. Disponible: http:// wwwasobanca.com [Consulta: 2012, Marzo 28].

Banco Occidental de Descuento (2012). Descripción de las funciones del área de Inteligencia de Negocios. Documentación Técnica del área de Inteligencia de Negocios. Maracaibo, Venezuela.

Davis, Jim; Miller, Gloria y Allan, Russell (2008). La Revolución de la Información. Editorial Profit. Madrid, España.

Guanipa, Mary y Ramírez, Paola (2011). Inteligencia de Negocios y Toma de Decisiones de los Gerentes en la Banca Universal en Venezuela. Revista CICAG. Año 2011, Volumen 8, Edición $\mathrm{N}^{\circ}$ 2. Maracaibo, Venezuela. pp. 50-63.

Herrera Osorio, Edward J. (2009). La Inteligencia de Negocios basada en el Proceso Unificado. Trabajo Especial de Grado. Universidad Nacional de Colombia. Bogotá D.C.

Hurtado, Fernando (2008). Tratamiento de las tecnologías de inteligencia de negocios (BI) en la estrategia de (TI). Revista Sistemas. Año 2008, N 106. Bogotá, Colombia. pp. 57-68.

Lluís, Joseph (2007). Business Intelligence: Competir con Información. Madrid, España. ESADE Business School. Pp. 392.

Oramas, Joaquín (2009). La inteligencia de negocios, un concepto informático. Revista Sistemas. Año 2009, N ${ }^{\circ}$ 111. Bogotá, Colombia. Pp. 42-51.

Olszak, Celina y Ziemba, Ewa (2012). Critical Success Factors for Implementing Business Intelligence Systems in Small and Medium Enterprises on the Example of Upper Silesia, Poland. Revista de la Universidad de Economía. Interdisciplinary Journal of Information, Knowledge, and Mana- 
gement. Año 2012, Volumen 7. Katowice, Polonia. pp. 129-150.

Peña Ayala, Alejandro (2006). Inteligencia de Negocios: Una Propuesta para su Desarrollo en las organizaciones. Publicación del Instituto Politécnico Nacional. México D.F.

Plant, Robert y Murrell, Stephen (2007). An executive's guide to information technology. Cambridge University Press. Primera Edición. U.K.

Pineda, O. y Díaz, C. (2006). Que hace que un proyecto de Business Intelligence sea exitoso. Publicación de la Uni- versidad EAFIT (Escuela de Administración, Finanzas y Tecnologías). Colombia.

Rainardi, Vincent (2007). Building a Data Warehouse: With Examples in SQL Server. Editorial Appress Academic. New York, USA.

Vitt Elizabeth; Luckevich, Michael y Misner, Stacia (2003). Business Intelligence, técnicas de análisis para la toma de decisiones estratégicas. Editorial Mc Graw Hill. Madrid, España. 